OPEN ACCESS

Edited by:

Jesus Avila,

Universidad Autonoma de Madrid, Spain

Reviewed by:

Gavin John Clowry,

Newcastle University,

United Kingdom

Tobias Engel,

Royal College of Surgeons in Ireland,

Ireland

${ }^{*}$ Correspondence:

Orly Reiner

orly.reiner@weizmann.ac.il

Received: 06 February 2018 Accepted: 16 April 2018

Published: 07 May 2018

Citation:

Gorelik A, Sapir T, Ben-Reuven L and Reiner O (2018) Complement C3 Affects Rac1 Activity in the

Developing Brain.

Front. Mol. Neurosci. 11:150. doi: 10.3389/fnmol.2018.00150

\section{Complement C3 Affects Rac1 Activity in the Developing Brain}

\author{
Anna Gorelik, Tamar Sapir, Lihi Ben-Reuven and Orly Reiner* \\ Department of Molecular Genetics, Weizmann Institute of Science, Rehovot, Israel
}

The complement system, which is part of the innate immune response system, has been recently shown to participate in multiple key processes in the developing brain. Here we aimed to elucidate downstream signaling responses linking complement C3, a key molecule of the pathway, to small GTPases, known to affect the cytoskeleton. The expression pattern of the activated small GTPase Rac1 resembled that of complement C3. C3-deficient mice exhibited reduced Rac1 and elevated RhoA activity in comparison with control mice. The most pronounced reduction of Rac1 activity occurred at embryonic day 14. Rac1 has been implicated in neuronal migration as well as neuronal stem cell proliferation and differentiation. Consistent with the reduction in Rac1 activity, the expression of phospho-cofilin, decreased in migrating neurons. Reduced Rac1-GTP was also correlated with a decrease in the expression of progenitor markers (Nestin, Pax6 and Tbr2) and conversely the expression of neuronal markers (Dcx and NeuN) increased in $\mathrm{C} 3$ knockout $(\mathrm{KO})$ cortices in comparison with wild-type (WT) cortices. More specifically, C3 deficiency resulted in a reduction in the number of the cells in S-phase and an elevation in the number of cells that precociously exited the cell cycle. Collectively, our findings suggest that C3 impacts the activity of small GTPases resulting in cell cycle defects and premature neuronal differentiation.

Keywords: complement C3, cortical development, neuronal stem cells, cell cycle, Rac1

\section{INTRODUCTION}

Complement acts as a rapid and efficient immune surveillance system that has distinct effects on healthy and altered host cells and foreign intruders (reviews Walport, 2001a,b; Zipfel et al., 2007; Ricklin et al., 2010; Hawksworth et al., 2017). The complement system is composed of a large family of proteins, which are either secreted or membrane bound. These proteins are usually inactive until the system is triggered by stimuli. Complement is activated by three major routes: the classical, the alternative and the lectin pathways, all of which converge on complement component $\mathrm{C} 3$, a central molecule in the system that ultimately drives complement effector functions, including the elimination of pathogens, debris and cellular structures. Several complement proteins are cleaved during activation of the system; for example, C3 is cleaved into two fragments, C3a and C3b.

During recent years, the notion that the immune system participates in regulation of complex behavior has emerged and it has been proposed to be malfunctioning in diseases as diverse as autism spectrum disorder (Hsiao et al., 2012; Onore et al., 2012), as well as late onset diseases such as Alzheimer's (review Veerhuis et al., 2011). Mutations in members of the lectin arm of the complement pathway have been previously implicated in 3MC syndrome (Degn et al., 2011; Rooryck et al., 2011), in which intellectual impairment is part of the complex syndrome. 
C3 regulates the number and function of glutamatergic synapses in the hippocampus and exerts negative effects on hippocampusdependent cognitive performance (Perez-Alcazar et al., 2014). C3 deficiency spared age dependent synaptic and neuronal loss in a region-specific manner and protected against cognitive impairment in normal aging of wild-type (WT) mice (Shi et al., 2015). Furthermore, it was shown that C3 deficiency is beneficial in Alzheimer's disease model as it protected against age- and plaque related synapse and neuron loss, decreased glial reactivity and spared cognitive decline in APP/PS1mice despite an increased plaque burden in the mouse brain (Shi et al., 2017). Innate immune molecules have been found participate in regulation of synaptic plasticity (review Boulanger and Shatz, 2004). The activity of the complement pathway has been implicated in developmental pruning of synapses refinement of the mouse visual system (Stevens et al., 2007; Schafer et al., 2012; Bialas and Stevens, 2013).

Our recent research has shown that immune signaling plays a role in early neural brain development, which includes neuronal stem cell proliferation and neuronal migration (Gorelik et al., 2017a,b). In particular, we have shown that key proteins in the lectin arm of this pathway, MASP1, MASP2 and C3, are expressed in the developing cortex and that neuronal stem cell proliferation and neuronal migration is affected in $\mathrm{KO}$ and knockdown mice. Molecular mimics of C3 cleavage products rescued the migration defects that have been seen following knockdown of C3 or Masp2. Pharmacological activation of the downstream receptors rescued Masp 2 and C3 knockdown as well as C3 KO (Gorelik et al., 2017a). An additional study investigated the complex developmental roles of Serping1 or C1 inhibitor, which is known to inhibit the initiation of the complement cascade (Gorelik et al., 2017b). Knockdown or KO of Serping1 affected neuronal stem cell proliferation and impaired neuronal migration in mice both in a cell-autonomous and non-cell autonomous manner. Most importantly, expression of protein components mimicking cleaved C3 rescued the knockdown of Serping1, indicating complement pathway functionality. Despite compelling evidence for the complement functions in the developing brain, the molecular mechanisms that allow these secreted molecules to exert their function is unknown. C3a and $\mathrm{C} 5$ a receptors are G-protein coupled, which are involved in a wide repertoire of cellular signaling. Activation of G-proteins may induce cytoskeletal rearrangement, which is required for cell division and radial neuronal migration. As key regulators of actin and microtubule cytoskeletons, cell polarity and adhesion, the Rho GTPases play critical roles in CNS neuronal migration (reviews Govek et al., 2011; Evsyukova et al., 2013). Furthermore, activation of the $\mathrm{C} 3 \mathrm{a}$ receptor by $\mathrm{C} 3 \mathrm{a}$ induced the activity of Rac1, a Rho GTPase, in migrating crest cells (CarmonaFontaine et al., 2011). Moreover, when Racl was inhibited, neural crest cell explants lost their coattraction, supporting the idea that this mechanism occurs by mutual chemoattraction, possibly via Rac1 activated by $\mathrm{C} 3 \mathrm{aR}$ upon binding to $\mathrm{C} 3 \mathrm{a}$. Rho family GTPases function as molecular switches and cycle between an active, GTP-bound state, and an inactive, GDP-bound state (review Iden and Collard, 2008). In this current study, we demonstrated the effect of C3 deficiency on the activity of small GTPases, in particular Rac1, and revealed how this affects neuronal stem cell cycle and cell fate determination.

\section{MATERIALS AND METHODS}

\section{Antibodies}

Mouse anti RAC1 (Millipore, 1:1000), rabbit anti RHOA (Cell Signaling, 1:1000), mouse anti CDC42 (Cytoskeleton, 1:1000), rabbit anti Glutathione-S-Transferase (GST; Santa Cruz, 1:2000) and rabbit anti EMERIN (Santa Cruz, Miami, FL, USA-254 $1: 1000)$ were used for western blotting.

The following antibodies were used for immunostainings: mouse anti RAC1 (Millipore, 1:200), mouse anti active RAC1 (NewEast Biosciences, 1:200), rabbit anti C3 (Antibody Verify, 1:400), mouse anti Phospho-COFILIN (Santa Cruz, 1:200), mouse anti iododeoxyuridine (IdU)-B44 (BD Biosciences, 1:200, 347580).

\section{Animals}

Animal protocols were approved by the Weizmann Institute IACUC and were carried out in accordance with their approved guidelines (approval number 34400317-2). C3 KO mice were obtained from the Jackson Laboratory. Male and female embryos were used in the study.

\section{Small GTPases Activation Assay}

GST-P21 activated kinase (GST-PAK), GST-RHOTEKIN (GST-RTKN) and GST were purified in NETN buffer $(0.5 \%$ NP-40, 20 mM Tris- $\mathrm{HCl}$ pH 8, $100 \mathrm{mM} \mathrm{NaCl}, 1 \mathrm{mM}$ EDTA) supplemented with PMSF and incubated with glutathioneagarose beads.

Cortices (two brains per tube) from C3 KO or WT embryos (E16) were collected on ice, immediately dissociated in the lysis buffer (50 mM Tris- $\mathrm{HCl} \mathrm{pH} \mathrm{7.5;} 150 \mathrm{mM} \mathrm{NaCl}$; 1 mM EDTA; 1 mM EGTA; 1\% Triton X-100 supplemented with aprotinin, leupeptin, $\mathrm{NaF}$, sodium orthovanadate and protease inhibitor cocktail) and centrifuged at 13,000 rpm. $0.5 \%$ of the resulted soup was used directly for western blot analysis to determine the general levels of small GTPases. The rest of the soup from each condition was divided to three equal parts and incubated overnight in $4^{\circ} \mathrm{C}$ with GST-PAK, GST-RTKN or GST treated beads. The beads were washed three times with NETN buffer. The pelleted beads were eluted by heating in $2 \times$ SDS-buffer. The samples were separated by SDS-PAGE and subjected to western blot analysis with the indicated antibodies. The relative quantification of the protein bands was performed with ImageJ "Gels" measurements. The intensity measurements of the bands of the small GTPases from the pull down were normalized to the intensity of the GST bands and to the level of the relevant small GTPase in the brain lysates. Emerin intensity served as the loading control of the lysates. The resulted data from six independent biological repeats of the WT and C3 KO were analyzed with the Student $t$-test. The averages are presented in the graphs. 


\section{IdU/EdU Labeling}

The thymidine analogs IdU $(0.01 \mathrm{ml}$ of $5 \mathrm{mg} / \mathrm{ml}$ IdU solution per gram body weight) and 5-ethynyl-2'-deoxyuridine (EdU, $50 \mathrm{mg}$ per gram body weight) were injected intraperitoneally to pregnant $\mathrm{C} 3 \mathrm{KO}$ and $\mathrm{WT}$ mice (E14) $3 \mathrm{~h}$ and $30 \mathrm{~min}$ respectively before sacrifice. The brains were removed and fixed in 2.5\% PFA-PBS overnight, washed and cryoprotected by immersion in $30 \%$ sucrose-PBS solution. The cryosections $(10 \mu \mathrm{m})$ were pretreated in boiling sodium citrate buffer (10 mM, pH 6) for $30 \mathrm{~min}$. The click reaction was performed with $\mathrm{Cy} 3$ azide $(2.5 \mu \mathrm{M})$ in the PBS-based buffer containing $100 \mathrm{mM}$ Tris- $\mathrm{HCl}, 1 \mathrm{mM} \mathrm{CuSO}_{4}$ and $100 \mathrm{mM}$ ascorbic acid. To block EdU epitopes before immunostaining with anti IdU antibodies the reaction was followed by a click reaction with a non-fluorescent molecule (Phenylthiomethyl-Azide $20 \mathrm{mM}$, Sigma). After treatment with $10 \mathrm{mM}$ ascorbic acid and $4 \mathrm{mM} \mathrm{CuSO}_{4}$, followed by incubation with $20 \mathrm{mM}$ EDTA, the immunostainings with anti-IdU-B44 were performed. The length of $S$ phase was calculated $\mathrm{T}_{\text {s-phase }}=\frac{T i}{L / S}$, given $\mathrm{i}=$ labeling interval $2.5 \mathrm{~h}$, $\mathrm{L}_{\text {(leaving) }}=\mathrm{Idu}^{+} \mathrm{EdU}^{-}, \mathrm{S}_{\text {(currently at S-phase })}=\mathrm{IdU}^{+} \mathrm{EdU}^{+}$. This protocol was adapted from a previously published procedure (Nowakowski et al., 1989). Overall, click reactions can be successfully combined with immunostainings (Kalveram et al., 2013).

\section{Immunocytochemistry}

Floating vibratome sections $(60 \mu \mathrm{m})$ or cryosections $(10 \mu \mathrm{m})$ were permeabilized using $0.1 \%$ Triton X-100 and blocked in blocking solution (PBS, $0.1 \%$ Triton X-100, 10\% HS; 10\% FBS) for $60 \mathrm{~min}$. Antibodies were incubated in blocking solution over night at $4^{\circ} \mathrm{C}$. After washing, appropriate secondary antibodies (Jackson ImmunoResearch) were diluted in blocking solution, and incubated for $2 \mathrm{~h}$ at room temperature. Slices were mounted onto glass slides using Aqua Polymount (Polysciences). Brains of embryos treated with EdU/IdU were fixed in 2.5\% PBS-PFA, cryoprotected in $20 \%$ sucrose and cryo-sectioned to $14 \mu \mathrm{m}$ thick slices before further processing.

\section{Microscopy, Quantification and Statistical Analyses}

Images were taken using confocal microscopy (LSM800 Zeiss), equipped with Axio Observer Z1 microscope, and imaged with either Plan-apochromat $20 \times / 0.8$, or Plan-apochromat $63 \times / 1.4$ oil objectives. The scaling data are $0.624 \times 0.624 \mu \mathrm{m}$ per pixel for $20 \times$ magnification, and $0.198 \times 0.198 \times 0.51 \mu \mathrm{m}$ per voxol for $60 \times$ magnification. The images were processed by ZEN software and/or Imaris software. Cell count and colocalization analyses were performed using Imaris software (Bitplane Inc., Zurich, Switzerland, Imaris core module). Three brains were analyzed for each treatment. Four representative slices from each brain were chosen for analysis. The size of the area of interest was determined and preserved per each experiment. For the cell counts the relevant channel of an area of interest was analyzed with "Spots" module of Imaris, every spot labeling approximate center of the cell body. For double-labeling the new channel was created with Imaris channel mixer and this channel was analyzed with "Spots" module. For the intensity analysis, the mean gray values (ImageJ) of the relevant channel of the identical areas of the cortex were compared. Comparisons of intensities were conducted using the whole width of the imaged cortex. Statistical analysis was performed by $t$-test or $t$-test with Bonferroni correction. Error bars represent standard error.

\section{Real-Time qRT-PCR}

E14 cortices (two brains for each repeat, $N=4$ ) from $C 3 \mathrm{KO}$ and WT were dissected in cold PBS and RNA isolation was performed according with Sigma protocol (TRI reagent, Sigma). After Dnase treatment (Sigma), first-strand cDNA synthesis was done using M-MLV RT (Promega). Relative levels of expression (three technical repeats for each sample) were normalized to the 29rps gene. Real-time PCR with SYBR FAST ABI qPCR kit (Kapa Biosystems) was performed using (Quant Studio 5). The following sets of primers were used for Real-time PCR reactions: 29rps: $5^{\prime}$-TCGTTGGGCGTCTGAAGGCAA and 5' -CGGAAG CACTGGCGGCACAT; C3aR: 5'-GGTGAGATGGAGGAACC AGA and 5'-ATTGGGACTGCTAGGCAATG; Nestin: 5'-GC AACTGGCACACCTCAAGA and $5^{\prime}$-AGCAGAGTCCTGTAT GTAGCC; Pax6: 5'-CTTTGAGAAGTGTGGGAACCAG and 5'-TGGTTAAAGTCTTCTGCCTGTGAG; Sox2: 5'-TTCGCA GGGAGTTCGCAAAA and 5'-ACCCAGCAAGAACCCTTT CC; Svet1: 5'-GTCGTAGCAACAGGATAGATGAG and 5'-GG CAAACCATTGGGAACTCGTG; NeuroD1: 5'-ACAACAGG AAGTGGAAACATGACC and $5^{\prime}$-CACTCATCTGTCCAGCT TGGG; Tbr2: 5'-GACCTCCAGGGACAATCTGA and 5'-GG CCTACCAAAACACGGATA; Dcx: 5'-GAGTGGGGCTTTCG AGTGAT and 5'-GGAACCACAGCAACTTTTCCAA; NeuN: $5^{\prime}$-GCGGAAACCTCCTCGGACAG and 5'-TTTTCAACGGG TTCAGCGTTCC; Satb2: 5'-CAGCCAGCCAAGTTTCAGAC and $5^{\prime}$-GGAATCATCAAACCTCCCACGG.

\section{RESULTS}

\section{The Activity of Small GTPases in C3 Brain Lysates}

As mentioned above, previous studies have demonstrated that $\mathrm{Racl}$ is activated following the binding of $\mathrm{C} 3 \mathrm{a}$ to the $\mathrm{C} 3 \mathrm{a}$ receptor (Carmona-Fontaine et al., 2011), therefore, we examined the activity of the small GTPases Rac1, RhoA and Cdc42 in the developing brains of $\mathrm{WT}$ and $\mathrm{C} 3 \mathrm{KO}$ mice (Figure 1). The activity of these proteins is regulated by the interaction of Rho family GTPases with guanine-nucleotide exchange factors (GEFs) and GTPase-activating proteins (GAPs). The activated small GTPases bind to their effectors. For example, the GTP-bound Rac1 bind to p21 protein (Cdc42/Rac)-activated kinase 1 (PAK) protein through its Cdc42-Rac-interactivebinding (CRIB) domain (Frost et al., 1997). This property enabled measuring the activity of the small GTPases in brain lysates using pull-down experiments with recombinant proteins expressing the binding domains (from PAK1 for Cdc42 and Racl and from Rhotekin (RTKN) for RhoA; Figure 1). The activity was determined by the ratio of the signal of active pulled-down proteins (Rac1, RhoA, or Cdc42) vs. the amount of 

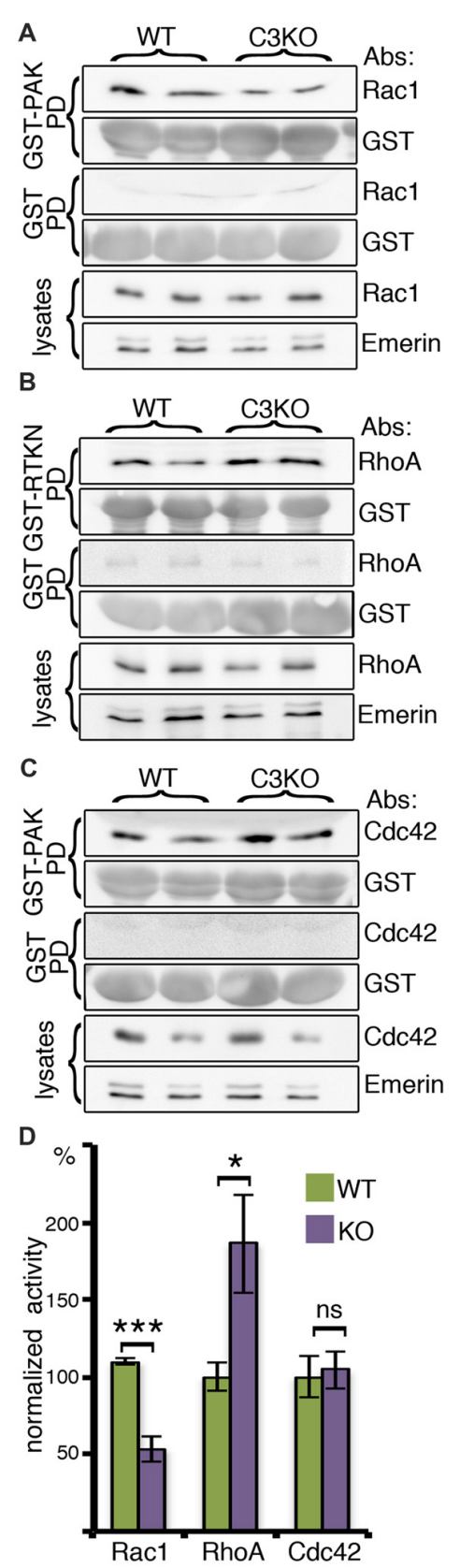

FIGURE 1 | The activity of the small Rho GTPases, RAC1, RHOA and CDC42, in brain lysates. Protein extracts from $\mathrm{C} 3$ knockout $(\mathrm{KO} ; N=6)$ and wild-type (WT; $N=6$ ) E16 cortices were incubated with glutathione-agarose beads pretreated with GST-P21 activated kinase

Glutathione-S-Transferase-P21 activated kinase (GST-PAK; A,C), GST-Rhotekin Rhotekin (RTKN; B) or GST as control for all conditions (A-C). The amount of activated RAC1 (A) was determined as a ratio of the amount of RAC1 bound to GST-PAK normalized to the amount of GST-PAK in the reaction and to the total amount of RAC1 in the brain lysate (RAC1/EMERIN in lysates). In a similar way, the activity of RHOA (B) and CDC42 was quantified (C). All three proteins did not bind to GST. (D) Comparison of the normalized activity is shown. Student's t-test, ${ }^{* * *} p<0.001,{ }^{*} p<0.05$, ns, $p=0.81$.

the relevant recombinant protein in reaction, normalized by the total amount of the small GTPases in the lysate (Figures 1A-C).
C3 KO mice exhibited a significant reduction in Rac1 activity and a significant elevation in RhoA activity, while no change was observed in the activity of Cdc42 (Figure 1D).

\section{In Situ Localization of Rac1-GTP}

To better understand how the reduction in Racl activity in $\mathrm{C} 3 \mathrm{KO}$ impacts brain development, immunostainings with anti-Rac1 and anti-C3 antibodies were conducted on E14 embryonic brain slices (Figures 2A,B). The distribution of the active Racl signal was most pronounced in the area which is in between the intermediate zone and the cortical plate, the subplate, and in the marginal zone. Prior to entering the cortical plate migrating neurons usually change their polarity (Tabata and Nakajima, 2003). Interestingly, immunostainings with anti-C3 antibodies demonstrate an increased signal in the same domain (Figure 2A). Higher magnification images reveal that in many cases the C3 signal is juxtaposed to active Rac1, which could be expected from an extracellular secreted molecule and an intracellular signaling molecule, respectively (Figure 2B). We next proceeded to examine whether the expression of total Rac1 differs from that of active Rac1. At E16, Racl is widely expressed in the developing brain and a more pronounced signal can be seen in the intermediate zone and the cortical plate. Active Racl strongest signal was observed in the subplate similar to E14 (Figure 2C). Then, we examined how active Racl is modulated in embryonic C3 KO brain (Figures 2D-G). At embryonic day 14 and 16, Rac1 activity was markedly and significantly reduced in C3 deficient brain sections (Figures 2D,E,G). Rac1 activity at embryonic day 18 did not differ between the $\mathrm{C} 3 \mathrm{KO}$ and the WT brains (Figures 2F,G). Rac1 has multiple downstream targets, amongst them it is known to induce the phosphorylation of Cofilin, which promotes actin polymerization (Delorme et al., 2007). Furthermore, previous studies have demonstrated that Cofilin is phosphorylated in migrating neurons, downstream to the Reelin pathway, and this activity is important to promote neuronal migration (Chai et al., 2009; Frotscher et al., 2017). Therefore, E16 brain sections were immunostained with anti-phosphoCofilin antibodies (Figure $\mathbf{2 H}$ ). The sections from $\mathrm{C} 3 \mathrm{KO}$ mice displayed significantly reduced levels of phospho-Cofilin, which mirrored the reduction in activated Rac1 (Figure 2I). Interestingly, the reduction in Racl activity was temporal and correlated well with the developmental peak in neuronal stem cell proliferation and neuronal migration. We speculate that at least part of the neuronal migration impairments observed in the developing C3 deficient mice are due to the reduction in active Racl and consequently phosphorylated Cofilin (Gorelik et al., 2017a).

\section{Rac1-GTP in the VZ and SVZ}

Next, the proliferative zones, which include the ventricular zone (VZ) and subVZ (SVZ) were examined more closely (Figure 3). Rac1-GTP is localized mainly to the apical membrane in the VZ (Figure 3A and insert), with some cells showing a signal in the cell soma (Figure 3A). Racl activity is significantly and markedly reduced in the same areas in C3 KO mice (Figures $\mathbf{3 A}, \mathbf{B}$ ). Reduced Racl activity within these stem cell niches may affect 


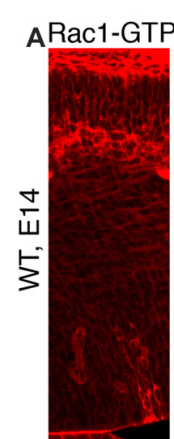

.

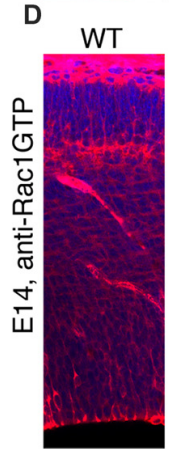

$\mathrm{H}$
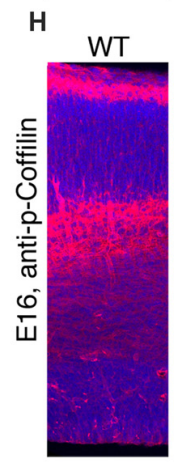
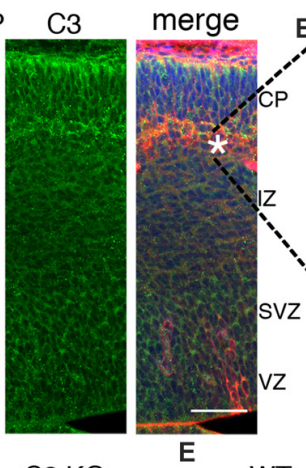

$\mathrm{C} 3 \mathrm{KO}$

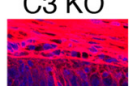

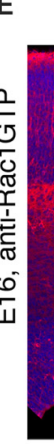

B
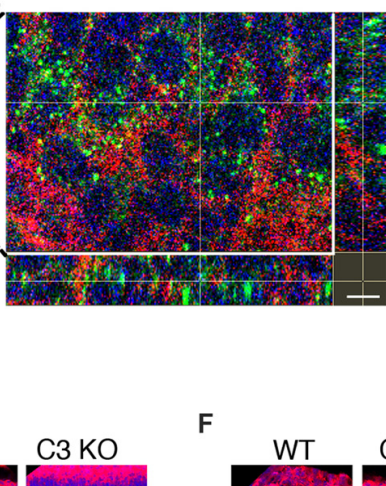

F<smiles>[CH][C]1CC[Al]1</smiles>

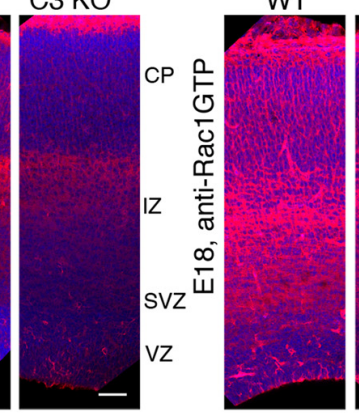

$\mathrm{C} 3 \mathrm{KO}$
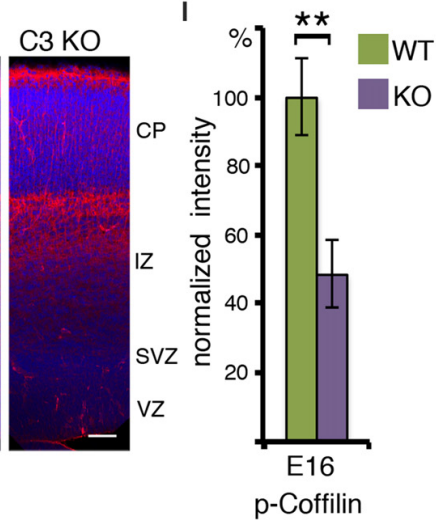
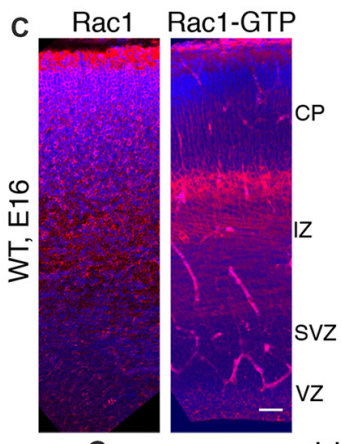

G

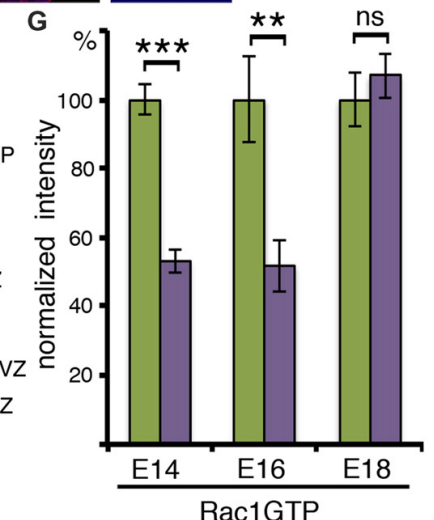

$\mathrm{C} 3 \mathrm{KO}$

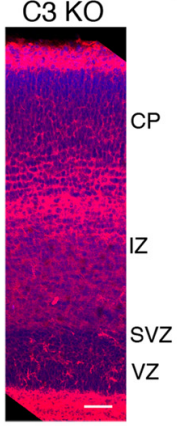

Rac1GTP

FIGURE 2 | Activated RAC1 in the developing cortex. (A) E14 brain sections were immunostained with anti-RAC1-GTP and anti-C3 antibodies. RAC1-GTP shows the highest intensity on the entrance to the cortical plate (CP). The scale bar is $50 \mu \mathrm{m}$. (B) High-magnification shows that in this area the signal of $\mathrm{C} 3$ is in close vicinity to that of RAC1-GTP. The scale bar is $5 \mu \mathrm{m}$. (C) E16 brain sections were immunostained with anti-RAC1 or anti-RAC1-GTP antibodies. Total RAC1 was evenly distributed all over the cortex, whereas the intensity of RAC1-GTP immunostaining signal was the highest between intermediate zone (IZ) and CP. The scale bar is $50 \mu \mathrm{m}$. (D-F) Comparison of anti-RAC1-GTP immunostaining in WT and C3 KO on different embryonic days: E14 (D, $N=6)$, E16 (E, $N=6)$, E18 (F, $N=6)$. (G) The normalized intensity of RAC1-GTP is presented as percentage of WT levels. (H) WT and C3 KO E16 brain sections ( $N=6$ ) were immunostained with anti-phospho-COFILIN antibodies. The scale bars are $50 \mu \mathrm{m}$. (I) The normalized intensity of phospho-COFILIN is presented as percentage of WT levels. Student's t-test, ${ }^{* * *} p<0.001,{ }^{* *} p<0.01$.

neuronal cell fate decision as well as cell cycle parameters. Therefore, we examined the steady state mRNA status of a battery of genes using RNA extracted from WT and C3 KO E14 cortices (Figure 3C). The expression of the $\mathrm{C} 3 \mathrm{a}$ receptor $\mathrm{C} 3 \mathrm{aR}$, increased in more than $25 \%$ in the $\mathrm{C} 3 \mathrm{KO}$, probably reflecting some compensatory pathways due to the absence of $\mathrm{C} 3$ and its cleavage products $\mathrm{C} 3 \mathrm{a}$ and $\mathrm{C} 3 \mathrm{~b}$. This finding may suggest that in $\mathrm{C} 3 \mathrm{KO}$ there may be alterations in other complement-related genes. Next we proceeded to investigate the expression of a battery of progenitor markers by means of real-time qPCR. A significant reduction was noted in the expression of two progenitor markers,
Nestin and Pax6. However, the expression of Sox2, which labels preferentially radial glia, but also intermediate progenitor cells (Hutton and Pevny, 2011) and Svet1, which labels multipolar cells in the SVZ (Tarabykin et al., 2001; Sasaki et al., 2008) did not vary between the WT and the control brains. In a similar manner, the expression of NeuroD1, which is highly expressed in the VZ, is upregulated several fold in the SVZ, and then is downregulated in the CP (Pataskar et al., 2016), was similar between the two genotypes. The expression of the intermediate progenitor marker, Tbr2 (Kowalczyk et al., 2009), was significantly reduced in the $\mathrm{C} 3 \mathrm{KO}$ mice. In a converse 

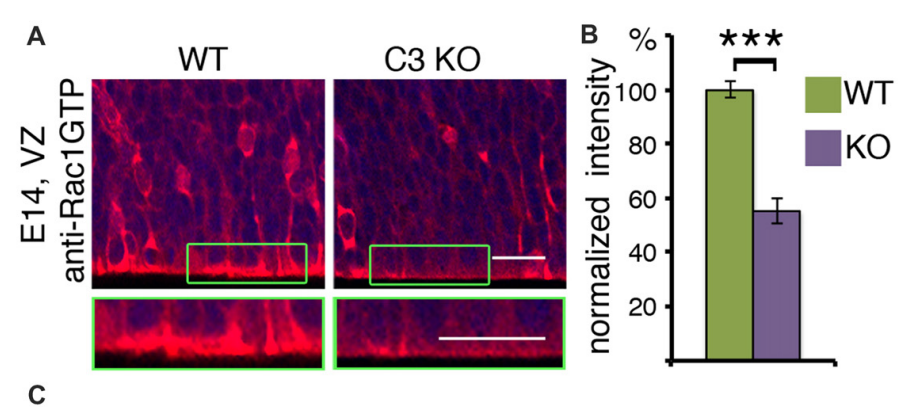

C
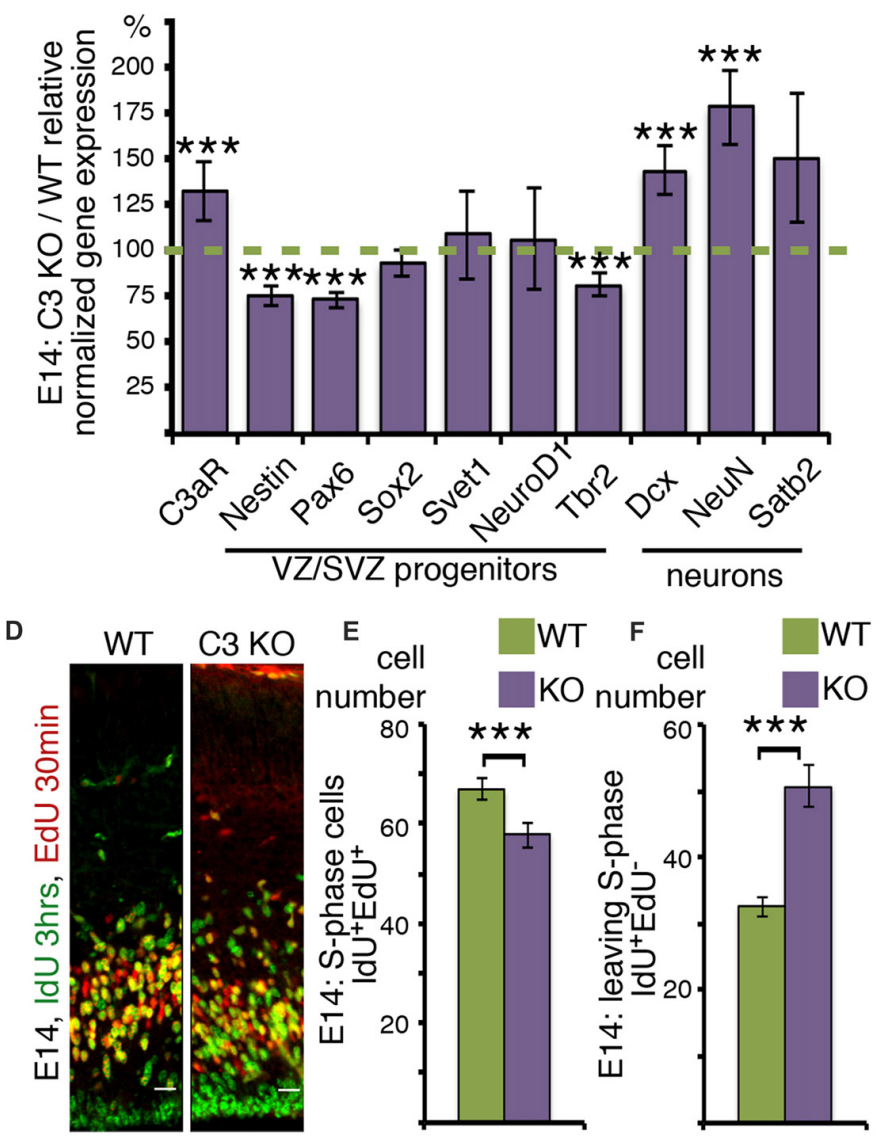

FIGURE 3 | C3 KO affects neuronal cell fate decisions and stem cell porliferation. (A,B) Ventricular zone (VZ) area of E14 WT and C3 KO brain sections ( $N=6$ ) immunostained with anti-RAC1-GTP antibodies. A higher magnification is shown in the green boxed insert. (B) The intensity of RAC1-GTP was measured and shown as percentage of WT levels. Student's $t$-test, ${ }^{* * *} p<0.001$ (C) Results of real-time qRT-PCR are presented as the ratio of mRNA expression levels of C3 KO to the WT. Two technical repeats of four brains for each genotype were used for analysis. Student's $t$-test with Bonferroni correction, ${ }^{* * *} p<0.001$. (D) $C 3 \mathrm{KO}$ and WT embryonic brains were labeled at E14 with IdU (3 h) and EdU (30 min). The brains were cryosectioned, EdU was detected by Cu(I)-catalyzed [3 + 2] cycloaddition reaction followed by immunostainings with anti-IdU antibodies. The scale bar is $20 \mu \mathrm{m}$. The number of cells per equal area which are actively in S-phase (IdU ${ }^{+}$EdU ${ }^{+}$ E) and leaving the cell cycle $\left(\mathrm{IdU}^{+} \mathrm{EdU}^{-} ; \mathbf{F}\right)$ compared between C3 KO $(N=17)$ and $\mathrm{WT}(N=22)$, Student's $t$-test, ${ }^{* * *} p<0.001$.

fashion, there was a significant increase in the expression of two early neuronal markers, Dcx (Francis et al., 1999) and NeuN (Mullen et al., 1992). Changes in the expression of the neuronal upper layer marker Satb2 (Britanova et al., 2008) were not significant. The observed decrease in some of the progenitor markers and the increase in some of the neuronal markers suggest that in $\mathrm{C} 3 \mathrm{KO}$ there may be a reduction in some of the neuronal progenitors which results in premature differentiation.
To gain a direct insight on cell cycle parameters, the progenitors were double labeled with two thymidine analogs, which are incorporated into the DNA during S-phase. The first label was done with IdU, followed by a second label with EdU. IdU was detected by immunostaining and EdU incorporation was visualized by click chemistry. The nuclei which are double labeled with both analogs either remained in S-phase for the duration of the combined labeling time or re-entered S-phase. The number of double positive cells decreased in the $\mathrm{C} 3 \mathrm{KO}$ 
mice (Figure 3E). Conversely, the number of cells that exited S-phase and were exclusively labeled by IdU, was higher in the C3 deficient embryos (Figure 3F). The duration of S-phase was $5.24 \pm 0.75 \mathrm{~h}$ in the WT animals and only $2.96 \pm 0.64 \mathrm{~h}$ in the $\mathrm{C} 3 \mathrm{KO}$ mice. In summary, C3 KO mice exhibited significant deviations in S-phase duration from the WT mice. Collectively, our study demonstrates that deficiency of C3 affected not only neuronal migration (Gorelik et al., 2017a) but also impaired cell cycle progression and the expression of cell fate markers.

\section{DISCUSSION}

Our current study suggests that the small GTPase Racl may be participating in mediating the signaling following complement activation in the developing brain. Small Rho GTPases are known to mediate cytoskeletal changes involving both the actin and the microtubule cytoskeleton, both of which are important for CNS development (reviews Govek et al., 2011; Evsyukova et al., 2013). Our study detected that C3 KO mice exhibit a significant reduction in Racl activity and a significant elevation in RhoA activity, while no change was observed in the activity of Cdc42. The reduction in Rac1 and elevation in RhoA fits with the known activities of these small GTPases. From the perspective of cell morphology, Racl and RhoA oppose each other. Canonical descriptions of cell migration place active Rac1 at the front of a migrating cell and active RhoA at its rear. Biochemically, Rac1 and RhoA are generally found to interact in mutually antagonistic ways, playing opposing roles in cell migration (review Guilluy et al., 2011). These opposing activities, as well as a negative feedback loop, lead to bistability in the signals regulating the dynamic cytoskeleton (Byrne et al., 2016). The availability of antibodies which recognize the active, GTP-bound form of Rac1 activation enabled the visualization of the temporal and spatial specific activity in the developing brain. Rac1 activity peaked at E14, coinciding with active neuronal migration and neuroblast proliferation. Migrating neurons entering the cortical plate demonstrated a strong Rac1-GTP signal which was reduced in C3 KO. The documented role of $\mathrm{C} 3$ in refinement of synaptic connections (Stevens et al., 2007) and the pattern of active Racl localization may point at additional and yet unexplored roles of C3 in early synaptogenesis. We found a particular high Rac1 GTP in the subplate, a transient zone that is located below the cortical plate and above the intermediate zone in the rodent developing cortex. The marginal zone and subplate neurons are among the first to be become post mitotic and they contribute to axon pathfinding and to circuits establishment (Balslev et al., 1996; López-Bendito and Molnár, 2003).

We further investigated the phosphorylation status of Cofilin, which is one of the downstream targets of Rac1. Cofilin is also known to promote neuronal migration and acts downstream to the Reelin pathway (Chai et al., 2009; Frotscher et al., 2017). The phosphorylation of Cofilin in C3 KO brains, was markedly reduced. Racl conditional $\mathrm{KO}$ mice exhibited delayed neuronal migration of pyramidal neurons (Chen et al., 2007). The phenotype observed in these Racl conditional KO mice was similar to that documented in $\mathrm{C} 3 \mathrm{KO}$, where neuronal migration was hindered but not completely halted (Gorelik et al., 2017a). Our previous studies have shown that the active lectin/MASP arm of the complement pathway, leading to cleavage of $\mathrm{C} 3$, and activating the complement peptide receptors for $\mathrm{C} 3 \mathrm{a}$ and $\mathrm{C} 5 \mathrm{a}$, is required for proper migration of neurons in the developing brain (Gorelik et al., 2017a). Furthermore, we suggested that functional activation of the pathway, resulting in $\mathrm{C} 3$ cleavage and production of $\mathrm{C} 3$ and $\mathrm{C} 5$ bioactive mediators, may be an important step in shaping the journey of neurons on their way to the cortical plate. Based on our current findings, we propose that Racl is one of the mediators of the complement signaling and that Racl facilitates transmission of the signal to the cytoskeleton, which will in turn mobilize the migrating neurons.

The possible effect on Racl activity was further investigated in the VZ and SVZ zones. A prominent signal of Rac1-GTP was noticed in the apical domain of neuroblasts in the VZ, which confirms previous studies (Minobe et al., 2009). In that study, addition of a Rac inhibitor or forced expression of a dominant-negative Racl significantly retarded interkinetic nuclear migration, and resulted in cytokinesis failures (Minobe et al., 2009). A role for Racl in the production of neuronal progenitors and cell fate specification was shown using a conditional allele (Chen et al., 2009). Rac1 deletion in the telencephalic VZ progenitors resulted in reduced sizes of both the striatum and cerebral cortex. Neuronal progenitors exhibited accelerated cell-cycle exit and increased apoptosis during early corticogenesis, leading to a decrease of the neural progenitor pool in mid-to-late telencephalic development (E16.5 to E18.5; Chen et al., 2009). Here, we have demonstrated a significant reduction in the length of $\mathrm{S}$-phase and an increased exit from the cell cycle in the $C 3$ deficient mice. We propose that complement $\mathrm{C} 3 \mathrm{KO}$ resulted in modified expression of some key progenitor genes. Recent studies demonstrate that at the single cell level there is a huge variability in gene expression levels, therefore, it is possible that the lack of C3 affects different progenitor populations in diverse manners. The expression of Nestin was significantly reduced following C3 KO; Nestin mRNA expression correlates with many, but not all, regions of proliferating CNS progenitor cells. In addition to its temporal and spatial regulation, Nestin expression is also regulated at the level of subcellular mRNA localization: in columnar neuroepithelial and radial glial cells Nestin mRNA was predominantly localized to the pial endfeet where most of the active Racl signal was localized to (Dahlstrand et al., 1995). In a similar manner, the expression of the key progenitor maker Pax6 was reduced in $\mathrm{C} 3 \mathrm{KO}$ brains. The levels of Pax6 are known to be essential for controlling the balance between neural stem cell self-renewal and neurogenesis (Sansom et al., 2009). However, the expression of Sox2 did not vary. Within the second population of progenitors, the intermediate progenitors, a reduction in the expression of the transcription factor Tbr2 (Kowalczyk et al., 2009), was noted, but no changes were noted in the expression of Svet1. One consequence of the reduced expression of some progenitor gene makers, shortening of S-phase and an increased exit from S-phase could be premature differentiation and depletion of the pool 
of progenitors. Indeed, we noted increased expression of the neuronal markers $D c x$ and $N e u N$, and in our previous studies we detected a reduction in the width of $\mathrm{C} 3 \mathrm{KO}$ cortices (Gorelik et al., 2017a). Collectively, our findings implicate Racl as one of the important downstream mediators of complement activity within the developing brain of the mouse embryo.

\section{AUTHOR CONTRIBUTIONS}

AG planned, conducted and analyzed most of the experiments. TS and LB-R planned, conducted and analyzed some of the experiments. OR planned and analyzed the experiments. All the authors participated in writing of the manuscript. All authors agree to be accountable for the content of the work.

\section{FUNDING}

The research has been supported by the Israel Science Foundation (Grant No. 347/15), the Legacy Heritage Biomedical

\section{REFERENCES}

Balslev, Y., Saunders, N. R., and Mollgard, K. (1996). Synaptogenesis in the neocortical anlage and early developing neocortex of rat embryos. Acta Anat. 156, 2-10. doi: 10.1159/000147822

Bialas, A. R., and Stevens, B. (2013). TGF- $\beta$ signaling regulates neuronal C1q expression and developmental synaptic refinement. Nat. Neurosci. 16, 1773-1782. doi: 10.1038/nn.3560

Boulanger, L. M., and Shatz, C. J. (2004). Immune signalling in neural development, synaptic plasticity and disease. Nat. Rev. Neurosci. 5, 521-531. doi: $10.1038 / \mathrm{nrn} 1428$

Britanova, O., de Juan Romero, C., Cheung, A., Kwan, K. Y., Schwark, M., Gyorgy, A., et al. (2008). Satb2 is a postmitotic determinant for upper-layer neuron specification in the neocortex. Neuron 57, 378-392. doi: 10.1016/j. neuron.2007.12.028

Byrne, K. M., Monsefi, N., Dawson, J. C., Degasperi, A., Bukowski-Wills, J. C., Volinsky, N., et al. (2016). Bistability in the Rac1, PAK, and RhoA signaling network drives actin cytoskeleton dynamics and cell motility switches. Cell Syst. 2, 38-48. doi: 10.1016/j.cels.2016.01.003

Carmona-Fontaine, C., Theveneau, E., Tzekou, A., Tada, M., Woods, M., Page, K. M., et al. (2011). Complement fragment C3a controls mutual cell attraction during collective cell migration. Dev. Cell 21, 1026-1037. doi: 10.1016/j.devcel.2011.10.012

Chai, X., Förster, E., Zhao, S., Bock, H. H., and Frotscher, M. (2009). Reelin stabilizes the actin cytoskeleton of neuronal processes by inducing $\mathrm{n}$-cofilin phosphorylation at serine3. J. Neurosci. 29, 288-299. doi: 10.1523/JNEUROSCI. 2934-08.2009

Chen, L., Liao, G., Waclaw, R. R., Burns, K. A., Linquist, D., Campbell, K., et al. (2007). Rac1 controls the formation of midline commissures and the competency of tangential migration in ventral telencephalic neurons. J. Neurosci. 27, 3884-3893. doi: 10.1523/JNEUROSCI.3509-06.2007

Chen, L., Melendez, J., Campbell, K., Kuan, C. Y., and Zheng, Y. (2009). Rac1 deficiency in the forebrain results in neural progenitor reduction and microcephaly. Dev. Biol. 325, 162-170. doi: 10.1016/j.ydbio.2008. 10.023

Dahlstrand, J., Lardelli, M., and Lendahl, U. (1995). Nestin mRNA expression correlates with the central nervous system progenitor cell state in many, but not all, regions of developing central nervous system. Dev. Brain Res. 84, 109-129. doi: 10.1016/0165-3806(94)00162-s

Degn, S. E., Jensenius, J. C., and Thiel, S. (2011). Disease-causing mutations in genes of the complement system. Am. J. Hum. Genet. 88, 689-705. doi: 10.1016/ j.ajhg.2011.05.011
Program of the Israel Science Foundation (Grant No. 2041/16), ERA-NET Neuron with support of the IMOH (Grant No. 3-0000-12276), by the European Cooperation on Science and Technology (COST Action CA16118), Weizmann-FAPESP supported by a research grant from Sergio and Sonia Lozinsky, Nella and Leon Benoziyo Center for Neurological Diseases, Jeanne and Joseph Nissim Foundation for Life Sciences Research, Wohl Biology Endowment Fund, Lulu P. and David J. Levidow Fund for Alzheimers Diseases and Neuroscience Research, the Helen and Martin Kimmel Stem Cell Research Institute, the Kekst Family Institute for Medical Genetics, the David and Fela Shapell Family Center for Genetic Disorders Research.

\section{ACKNOWLEDGMENTS}

We are grateful for the help of Ofira Higfa, Yehuda Melamed, Osnat Amram and $\mathrm{Oz}$ Yirmiyahu from the Weizmann Institute of Science. OR is the incumbent of the Bernstein-Mason Chair of Neurochemistry. TS is an Incumbent of the Leir Research Fellow Chair in Autism Spectrum Disorder Research.

Delorme, V., Machacek, M., DerMardirossian, C., Anderson, K. L., Wittmann, T. Hanein, D., et al. (2007). Cofilin activity downstream of Pak1 regulates cell protrusion efficiency by organizing lamellipodium and lamella actin networks. Dev. Cell 13, 646-662. doi: 10.1016/j.devcel.2007.08.011

Evsyukova, I., Plestant, C., and Anton, E. S. (2013). Integrative mechanisms of oriented neuronal migration in the developing brain. Annu. Rev. Cell Dev. Biol. 29, 299-353. doi: 10.1146/annurev-cellbio-101512-122400

Francis, F., Koulakoff, A., Boucher, D., Chafey, P., Schaar, B., Vinet, M. C., et al. (1999). Doublecortin is a developmentally regulated, microtubuleassociated protein expressed in migrating and differentiating neurons. Neuron 23, 247-256. doi: 10.1016/s0896-6273(00)80777-1

Frost, J. A., Steen, H., Shapiro, P., Lewis, T., Ahn, N., Shaw, P. E., et al. (1997). Cross-cascade activation of ERKs and ternary complex factors by Rho family proteins. EMBO J. 16, 6426-6438. doi: 10.1093/emboj/16. 21.6426

Frotscher, M., Zhao, S., Wang, S., and Chai, X. (2017). Reelin signaling inactivates cofilin to stabilize the cytoskeleton of migrating cortical neurons. Front. Cell. Neurosci. 11:148. doi: 10.3389/fncel.2017.00148

Gorelik, A., Sapir, T., Haffner-Krausz, R., Olender, T., Woodruff, T. M., and Reiner, O. (2017a). Developmental activities of the complement pathway in migrating neurons. Nat. Commun. 8:15096. doi: 10.1038/ncomms 15096

Gorelik, A., Sapir, T., Woodruff, T. M., and Reiner, O. (2017b). Serping1/C1 inhibitor affects cortical development in a cell autonomous and non-cell autonomous manner. Front. Cell. Neurosci. 11:169. doi: 10.3389/ fncel.2017.00169

Govek, E. E., Hatten, M. E., and Van Aelst, L. (2011). The role of Rho GTPase proteins in CNS neuronal migration. Dev. Neurobiol. 71, 528-553. doi: 10.1002/dneu.20850

Guilluy, C., Garcia-Mata, R., and Burridge, K. (2011). Rho protein crosstalk: another social network? Trends Cell Biol. 21, 718-726. doi: 10.1016/j.tcb.2011. 08.002

Hawksworth, O. A., Coulthard, L. G., and Woodruff, T. M. (2017). Complement in the fundamental processes of the cell. Mol. Immunol. 84, 17-25. doi: 10.1016/j. molimm.2016.11.010

Hsiao, E. Y., McBride, S. W., Chow, J., Mazmanian, S. K., and Patterson, P. H. (2012). Modeling an autism risk factor in mice leads to permanent immune dysregulation. Proc. Natl. Acad. Sci. U S A 109, 12776-12781. doi: 10.1073/pnas. 1202556109

Hutton, S. R., and Pevny, L. H. (2011). SOX2 expression levels distinguish between neural progenitor populations of the developing dorsal telencephalon. Dev. Biol. 352, 40-47. doi: 10.1016/j.ydbio.2011.01.015 
Iden, S., and Collard, J. G. (2008). Crosstalk between small GTPases and polarity proteins in cell polarization. Nat. Rev. Mol. Cell Biol. 9, 846-859. doi: 10.1038/ nrm2521

Kalveram, B., Lihoradova, O., Indran, S. V., Head, J. A., and Ikegami, T. (2013). Using click chemistry to measure the effect of viral infection on host-cell RNA synthesis. J. Vis. Exp. 78:e50809. doi: 10.3791/50809

Kowalczyk, T., Pontious, A., Englund, C., Daza, R. A., Bedogni, F., Hodge, R., et al. (2009). Intermediate neuronal progenitors (basal progenitors) produce pyramidal-projection neurons for all layers of cerebral cortex. Cereb. Cortex 19, 2439-2450. doi: 10.1093/cercor/bhn260

López-Bendito, G., and Molnár, Z. (2003). Thalamocortical development: how are we going to get there? Nat. Rev. Neurosci. 4, 276-289. doi: 10.1038/nrn1075

Minobe, S., Sakakibara, A., Ohdachi, T., Kanda, R., Kimura, M., Nakatani, S., et al. (2009). Rac is involved in the interkinetic nuclear migration of cortical progenitor cells. Neurosci. Res. 63, 294-301. doi: 10.1016/vj.neures.2009.01.006

Mullen, R. J., Buck, C. R., and Smith, A. M. (1992). NeuN, a neuronal specific nuclear protein in vertebrates. Development 116, 201-211.

Nowakowski, R. S., Lewin, S. B., and Miller, M. W. (1989). Bromodeoxyuridine immunohistochemical determination of the lengths of the cell cycle and the DNA-synthetic phase for an anatomically defined population. J. Neurocytol. 18, 311-318. doi: 10.1007/bf01190834

Onore, C., Careaga, M., and Ashwood, P. (2012). The role of immune dysfunction in the pathophysiology of autism. Brain Behav. Immun. 26, 383-392. doi: 10.1016/j.bbi.2011.08.007

Pataskar, A., Jung, J., Smialowski, P., Noack, F., Calegari, F., Straub, T., et al. (2016). NeuroD1 reprograms chromatin and transcription factor landscapes to induce the neuronal program. EMBO J. 35, 24-45. doi: 10.15252/embj.201591206

Perez-Alcazar, M., Daborg, J., Stokowska, A., Wasling, P., Björefeldt, A., Kalm, M., et al. (2014). Altered cognitive performance and synaptic function in the hippocampus of mice lacking C3. Exp. Neurol. 253, 154-164. doi: 10.1016/j. expneurol.2013.12.013

Ricklin, D., Hajishengallis, G., Yang, K., and Lambris, J. D. (2010). Complement: a key system for immune surveillance and homeostasis. Nat. Immunol. 11, 785-797. doi: 10.1038/ni.1923

Rooryck, C., Diaz-Font, A., Osborn, D. P., Chabchoub, E., HernandezHernandez, V., Shamseldin, H., et al. (2011). Mutations in lectin complement pathway genes COLEC11 and MASP1 cause 3MC syndrome. Nat. Genet. 43, 197-203. doi: 10.1038/ng.757

Sansom, S. N., Griffiths, D. S., Faedo, A., Kleinjan, D. J., Ruan, Y., Smith, J., et al. (2009). The level of the transcription factor Pax6 is essential for controlling the balance between neural stem cell self-renewal and neurogenesis. PLoS Genet. 5:e1000511. doi: 10.1371/journal.pgen.1000511

Sasaki, S., Tabata, H., Tachikawa, K., and Nakajima, K. (2008). The cortical subventricular zone-specific molecule Svet1 is part of the nuclear RNA coded by the putative netrin receptor gene Unc5d and is expressed in multipolar migrating cells. Mol. Cell. Neurosci. 38, 474-483. doi: 10.1016/j.mcn.2008. 04.002

Schafer, D. P., Lehrman, E. K., Kautzman, A. G., Koyama, R., Mardinly, A. R., Yamasaki, R., et al. (2012). Microglia sculpt postnatal neural circuits in an activity and complement-dependent manner. Neuron 74, 691-705. doi: 10.1016/j.neuron.2012.03.026

Shi, Q., Chowdhury, S., Ma, R., Le, K. X., Hong, S., Caldarone, B. J., et al. (2017). Complement C3 deficiency protects against neurodegeneration in aged plaque-rich APP/PS1 mice. Sci. Transl. Med. 9:eaaf6295. doi: 10.1126/ scitranslmed.aaf6295

Shi, Q., Colodner, K. J., Matousek, S. B., Merry, K., Hong, S., Kenison, J. E., et al. (2015). Complement C3-deficient mice fail to display age-related hippocampal decline. J. Neurosci. 35, 13029-13042. doi: 10.1523/JNEUROSCI.1698-15.2015

Stevens, B., Allen, N. J., Vazquez, L. E., Howell, G. R., Christopherson, K. S., Nouri, N., et al. (2007). The classical complement cascade mediates CNS synapse elimination. Cell 131, 1164-1178. doi: 10.1016/j.cell.2007.10.036

Tabata, H., and Nakajima, K. (2003). Multipolar migration: the third mode of radial neuronal migration in the developing cerebral cortex. J. Neurosci. 23, 9996-10001. doi: 10.1523/JNEUROSCI.23-31-09996.2003

Tarabykin, V., Stoykova, A., Usman, N., and Gruss, P. (2001). Cortical upper layer neurons derive from the subventricular zone as indicated by Svetl gene expression. Development 128, 1983-1993.

Veerhuis, R., Nielsen, H. M., and Tenner, A. J. (2011). Complement in the brain. Mol. Immunol. 48, 1592-1603. doi: 10.1016/j.molimm.2011.04.003

Walport, M. J. (2001a). Complement. First of two parts. N. Engl. J. Med. 344, 1058-1066. doi: 10.1056/NEJM200104053441406

Walport, M. J. (2001b). Complement. Second of two parts. N. Engl. J. Med. 344, 1140-1144. doi: 10.1056/NEJM200104123441506

Zipfel, P. F., Wurzner, R., and Skerka, C. (2007). Complement evasion of pathogens: common strategies are shared by diverse organisms. Mol. Immunol. 44, 3850-3857. doi: 10.1016/j.molimm.2007.06.149

Conflict of Interest Statement: The authors declare that the research was conducted in the absence of any commercial or financial relationships that could be construed as a potential conflict of interest.

Copyright (C) 2018 Gorelik, Sapir, Ben-Reuven and Reiner. This is an open-access article distributed under the terms of the Creative Commons Attribution License (CC BY). The use, distribution or reproduction in other forums is permitted, provided the original author(s) and the copyright owner are credited and that the original publication in this journal is cited, in accordance with accepted academic practice. No use, distribution or reproduction is permitted which does not comply with these terms. 